\title{
Catalytic Asymmetric Simmons-Smith Cyclopropanation of Silyl Enol Ethers. Efficient Synthesis of Optically Active Cyclopropanol Derivatives
}

\author{
Haifeng Du, Jiang Long, and Yian Shi*
}

Department of Chemistry

Colorado State University

Fort Collins, CO 80523

Supporting Information 


\section{Spectroscopic data for dipeptide 2}<smiles>CC(C)C(NC(=O)OCc1ccccc1)C(=O)N1CCCC1C(=O)c1ccccc1</smiles>

$[\alpha]^{20}=-69.9\left(c 1.65, \mathrm{CHCl}_{3}\right) ;$ IR (neat) $3310,1698,1638 \mathrm{~cm}^{-1} ;{ }^{1} \mathrm{H}$ NMR $\left(300 \mathrm{MHz}, \mathrm{CDCl}_{3}\right) \delta$ $8.00(\mathrm{dd}, J=7.2,1.5 \mathrm{~Hz}, 2 \mathrm{H}), 7.61-7.56(\mathrm{~m}, 1 \mathrm{H}), 7.47(\mathrm{t}, J=7.5 \mathrm{~Hz}, 2 \mathrm{H}), 5.56(\mathrm{dd}, J=8.7,5.1$

$\mathrm{Hz}, 1 \mathrm{H}), 5.21(\mathrm{~d}, J=9.3 \mathrm{~Hz}, 1 \mathrm{H}), 4.35(\mathrm{dd}, J=9.3,6.0 \mathrm{~Hz}, 1 \mathrm{H}), 3.93-3.85(\mathrm{~m}, 1 \mathrm{H}), 3.81-3.71$ $(\mathrm{m}, 1 \mathrm{H}), 2.38-2.26(\mathrm{~m}, 1 \mathrm{H}), 2.16-2.02(\mathrm{~m}, 3 \mathrm{H}), 2.00-1.88(\mathrm{~m}, 1 \mathrm{H}), 1.44(\mathrm{~s}, 9 \mathrm{H}), 1.09(\mathrm{~d}, J=6.9$ $\mathrm{Hz}, 3 \mathrm{H}), 0.96(\mathrm{~d}, J=6.9 \mathrm{~Hz}, 3 \mathrm{H}) ;{ }^{13} \mathrm{C} \mathrm{NMR}\left(75 \mathrm{MHz}, \mathrm{CDCl}_{3}\right) \delta$ 197.6, 170.7, 155.8, 132.2, 133.5, 128.7, 128.4, 79.4, 61.2, 56.9, 47.5, 31.5, 29.3, 28.5, 25.2, 19.6, 17.6; Anal. Cacld. for $\mathrm{C}_{21} \mathrm{H}_{30} \mathrm{~N}_{2} \mathrm{O}_{4}$ : C, 67.35; H, 8.07; N, 7.48; Found: C, 67.50; H, 8.01; N, 7.51.

\section{Representative catalytic asymmetric Simmons-Smith cyclopropanation and ligand recovery procedure (Table 1, Entry 1).}

To a flame-dried and Ar-filled $5 \mathrm{~mL}$-vial (Vial A) was added a solution of dipeptide 2 (112.4 mg, $0.30 \mathrm{mmol}, 0.25$ eq.) in freshly distilled $\mathrm{CH}_{2} \mathrm{Cl}_{2}(1.0 \mathrm{~mL})$, followed by the addition of neat $\mathrm{ZnEt}_{2}(37.0 \mathrm{mg}, 31 \mu \mathrm{L}, 0.30 \mathrm{mmol}, 0.25$ eq.). The solution was stirred at room temperature for $1 \mathrm{~h}$. Upon cooling to $0{ }^{\circ} \mathrm{C}$ under $\mathrm{Ar}, \mathrm{CH}_{2} \mathrm{I}_{2}(80.4 \mathrm{mg}, 24 \mu \mathrm{L}, 0.30 \mathrm{mmol}, 0.25$ eq.) was added dropwise, and then the solution was stirred at $0{ }^{\circ} \mathrm{C}$ for $0.5 \mathrm{~h}$.

Simultaneously, to another flame-dried and Ar-filled $5 \mathrm{~mL}$-vial (Vial B) was added freshly distilled $\mathrm{CH}_{2} \mathrm{Cl}_{2}(1.0 \mathrm{~mL})$, followed by neat $\mathrm{ZnEt}_{2}(148.2 \mathrm{mg}, 123 \mu \mathrm{L}, 1.2 \mathrm{mmol}, 1.0$ eq.). Upon cooling to $-78{ }^{\circ} \mathrm{C}$ under $\mathrm{Ar}, \mathrm{CH}_{2} \mathrm{I}_{2}(642.8 \mathrm{mg}, 193 \mu \mathrm{L}, 2.4 \mathrm{mmol}, 2.0$ eq.) was added dropwise. After the resulting mixture was stirred at this temperature for $1 \mathrm{~h}$ (a white precipitate formed), ethyl methoxyacetate (EMA) (141.8 mg, $141 \mu \mathrm{L}, 1.2 \mathrm{mmol}, 1.0$ eq.) was added. The 
reaction mixture was warmed to $-50{ }^{\circ} \mathrm{C}$ and a homogeneous solution was formed, the reaction mixture was then cooled to $-78^{\circ} \mathrm{C}$.

To the third flame-dried and Ar-filled $5 \mathrm{~mL}$-vial (Vial C) was added $\mathrm{I}_{2}(152.4 \mathrm{mg}, 0.6$ mmol, 0.5 eq.). Vacuum was applied for 10 seconds and then $\mathrm{CH}_{2} \mathrm{Cl}_{2}(0.5 \mathrm{~mL})$ was added. After the mixture was cooled to $0{ }^{\circ} \mathrm{C}$, neat $\mathrm{ZnEt}_{2}(37.0 \mathrm{mg}, 31 \mu \mathrm{L}, 0.30 \mathrm{mmol}, 0.25$ eq.) was added. Upon stirring at $0{ }^{\circ} \mathrm{C}$ for $1 \mathrm{~h}$, the reaction mixture was cooled to $-78{ }^{\circ} \mathrm{C}$ under Ar. The contents of Vial A and Vial B were then transferred to vial $\mathbf{C}$ via a cannula respectively, followed by the addition of (Z)-1-phenyl-1-(trimethylsilyloxy)-1-propene (247.5 mg, $1.2 \mathrm{mmol}$, 1.0 eq.). Upon warming to $-40{ }^{\circ} \mathrm{C}$ and stirring at this temperature for $48 \mathrm{~h}$, the reaction mixture was poured into hexanes $(100 \mathrm{~mL})$ and filtered. The filtrate was concentrated and purified by chromatography ( silica gel, hexanes:ether $=6: 1, \mathrm{v} / \mathrm{v}$ ) to afford the cyclopropane as colorless liquid (261.0 mg, 99\% yield, 93\% ee).

The above filter cake was dissolved in ethyl acetate $(25 \mathrm{~mL})$, and saturated aq. $\mathrm{NH}_{4} \mathrm{Cl}(20$ $\mathrm{mL}$ ) was added. The resulted mixture was stirred at room temperature for $30 \mathrm{~min}$, and the organic layer was separated. The aqueous layer was extracted with ethyl acetate $(25 \mathrm{~mL})$. The combined organics were dried $\left(\mathrm{Na}_{2} \mathrm{SO}_{4}\right)$, filtered, concentrated, and purified by flash chromatography ( silica gel, hexanes:ether $=2: 1, \mathrm{v} / \mathrm{v}$ ) to give dipeptide $\mathbf{2}$ as white sticky solid (101.3 mg, 90\%).

\section{Table 1, Entry 1}

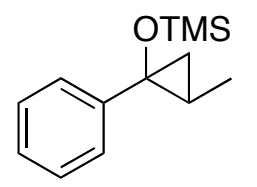


Colorless liquid; $[\alpha]_{\mathrm{D}}^{20}=+52.7\left(c 1.07, \mathrm{CHCl}_{3}\right)\left(93 \%\right.$ ee); IR (neat) 1603, 1450, $1251 \mathrm{~cm}^{-1} ;{ }^{1} \mathrm{H}$ NMR (300 MHz, $\left.\mathrm{CDCl}_{3}\right)$ d 7.32-7.23 (m, 4H), 7.20-7.16 (m, 1H), $1.34(\mathrm{dd}, J=9.6,5.4 \mathrm{~Hz}, 1 \mathrm{H})$, $1.25(\mathrm{~d}, J=5.7 \mathrm{~Hz}, 3 \mathrm{H}), 1.04-0.93(\mathrm{~m}, 1 \mathrm{H}), 0.76(\mathrm{t}, J=6.6 \mathrm{~Hz}, 1 \mathrm{H}), 0.10(\mathrm{~s}, 9 \mathrm{H}) ;{ }^{13} \mathrm{C} \mathrm{NMR}(75$ $\left.\mathrm{MHz}, \mathrm{CDCl}_{3}\right) \delta 145.9,128.1,126.0,124.8,61.1,23.2,22.4,13.2,1.5$.

Lorenz, J. C.; Long, J.; Yang, Z.; Xue, S.; Xie, Y.; Shi, Y. J. Org. Chem. 2004, 69, 327.

Table 1, Entry 2<smiles>COc1ccc(C2(O[Na])C(C)C2C)cc1</smiles>

Colorless liquid; $[\alpha]_{\mathrm{D}}^{20}=+44.3\left(c 1.26, \mathrm{CHCl}_{3}\right)\left(93 \%\right.$ ee); IR (neat) $1611,1514 \mathrm{~cm}^{-1} ;{ }^{1} \mathrm{H} \mathrm{NMR}$ $\left(300 \mathrm{MHz}, \mathrm{CDCl}_{3}\right) \delta$ 7.22-7.17 (m, 2H), 6.86-6.81 (m, 2H), $3.80(\mathrm{~s}, 3 \mathrm{H}), 1.25$ (dd, $J=9.6,5.4$ $\mathrm{Hz}, 1 \mathrm{H}), 1.24(\mathrm{~d}, J=6.0 \mathrm{~Hz}, 3 \mathrm{H}), 1.02-0.88(\mathrm{~m}, 1 \mathrm{H}), 0.67(\mathrm{t}, J=6.1 \mathrm{~Hz}, 1 \mathrm{H}), 0.07(\mathrm{~s}, 9 \mathrm{H}) ;{ }^{13} \mathrm{C}$ NMR $\left(75 \mathrm{MHz}, \mathrm{CDCl}_{3}\right) \delta 158.1,137.8,126.7,113.5,61.1,55.4,22.0,21.7,13.2,1.4$; Anal. Cacld. for $\mathrm{C}_{14} \mathrm{H}_{22} \mathrm{O}_{2} \mathrm{Si}$ : C, 67.15; H, 8.86; Found: C, 67.26; H, 8.96.

Kirihara, M.; Kakuda, H.; Tsunooka, M.; Shimajiri, A.; Takuwa, T.; Hatano, A. Tetrahedron Lett. 2003, 44, 8513.

\section{Table 1, Entry 3}

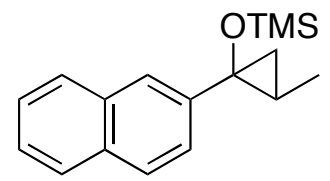

Colorless liquid; $[\alpha]^{20}{ }_{\mathrm{D}}=+67.4\left(c\right.$ 1.04, $\left.\mathrm{CHCl}_{3}\right)(95 \%$ ee $)$; IR (neat) $1251 \mathrm{~cm}^{-1} ;{ }^{1} \mathrm{H}$ NMR $(300$ $\left.\mathrm{MHz}, \mathrm{CDCl}_{3}\right) \delta$ 7.82-7.69 (m, 4H), 7.49-7.40 (m, 2H), $7.36(\mathrm{dd}, J=8.7,1.8 \mathrm{~Hz}, 1 \mathrm{H}), 1.49(\mathrm{dd}, J$ $=9.6,6.0 \mathrm{~Hz}, 1 \mathrm{H}), 1.30(\mathrm{~d}, J=6.0 \mathrm{~Hz}, 3 \mathrm{H}), 1.14-1.02(\mathrm{~m}, 1 \mathrm{H}), 0.83(\mathrm{t}, J=6.0 \mathrm{~Hz}, 1 \mathrm{H}), 0.12(\mathrm{~s}$, 
$9 \mathrm{H}) ;{ }^{13} \mathrm{C} \mathrm{NMR}\left(75 \mathrm{MHz}, \mathrm{CDCl}_{3}\right) \delta 143.4,133.3,132.1,127.9,127.6,126.1,125.4,123.9,123.0$, $61.4,23.1,22.3,13.2,1.5$.

Kirihara, M.; Kakuda, H.; Tsunooka, M.; Shimajiri, A.; Takuwa, T.; Hatano, A. Tetrahedron Lett. 2003, 44, 8513.

Table 1, Entry 4<smiles>CC1CCc2ccccc2C1(C)[O+]</smiles>

Colorless liquid; $[\alpha]^{20}{ }_{\mathrm{D}}=-32.5\left(c\right.$ 1.01, $\left.\mathrm{CHCl}_{3}\right)\left(93 \%\right.$ ee); IR (neat) $1488 \mathrm{~cm}^{-1} ;{ }^{1} \mathrm{H}$ NMR (300 $\left.\mathrm{MHz}, \mathrm{CDCl}_{3}\right) \delta 7.64(\mathrm{~d}, J=7.8 \mathrm{~Hz}, 1 \mathrm{H}), 7.24(\mathrm{t}, J=7.2 \mathrm{~Hz}, 1 \mathrm{H}), 7.11(\mathrm{t}, J=7.2 \mathrm{~Hz}, 1 \mathrm{H}), 7.05$ $(\mathrm{d}, J=7.2 \mathrm{~Hz}, 1 \mathrm{H}), 2.64(\mathrm{dd}, J=16.5,4.8 \mathrm{~Hz}, 1 \mathrm{H}), 2.40(\mathrm{td}, J=13.5,6.3 \mathrm{~Hz}, 1 \mathrm{H}), 2.07-2.00(\mathrm{~m}$, $1 \mathrm{H}), 1.85-1.73(\mathrm{~m}, 2 \mathrm{H}), 1.18(\mathrm{dd}, J=9.6,5.7 \mathrm{~Hz}, 1 \mathrm{H}), 1.02(\mathrm{t}, J=5.7 \mathrm{~Hz}, 1 \mathrm{H}), 0.15(\mathrm{~s}, 9 \mathrm{H}) ;{ }^{13} \mathrm{C}$ $\operatorname{NMR}\left(75 \mathrm{MHz}, \mathrm{CDCl}_{3}\right) \delta 141.1,132.4,128.2,126.2,125.3,125.1,56.2,26.4,24.0,18.7,16.5$, 1.5; Anal. Cacld. for $\mathrm{C}_{14} \mathrm{H}_{20} \mathrm{OSi}$ : C, 72.36; H, 8.67; Found: C, 72.34; H, 8.41.

(a) Rubottom, G. M.; Lopez, M. I.; J. Org. Chem. 1973, 38, 2097. (b) Murai, S.; Aya, T.; Sonada, N. J. Org. Chem. 1973, 38, 4354. (c) Ryu, I.; Aya, T.; Otani, S.; Sonode, N. J. Organomet. Chem. 1987, 321, 279.

\section{Table 1, Entry 5}

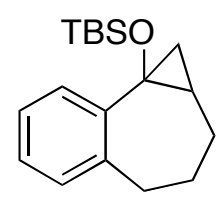

Colorless liquid; $[\alpha]^{20}{ }_{\mathrm{D}}=+9.8\left(c 0.98, \mathrm{CHCl}_{3}\right)\left(96 \%\right.$ ee); IR (neat) $1472,1221 \mathrm{~cm}^{-1} ;{ }^{1} \mathrm{H} \mathrm{NMR}$ $\left(300 \mathrm{MHz}, \mathrm{CDCl}_{3}\right) \delta$ 7.40-7.37 (m, 1H), 7.24-7.16 (m, 2H), 7.10-7.07 (m, 1H), 3.37 (td, $J=12.6$, $7.2 \mathrm{~Hz}, 1 \mathrm{H}), 2.55(\mathrm{dd}, J=12.9,6.3 \mathrm{~Hz}, 1 \mathrm{H}), 1.99-1.81(\mathrm{~m}, 2 \mathrm{H}), 1.57-1.44(\mathrm{~m}, 1 \mathrm{H}), 1.14(\mathrm{dd}, J=$ 9.6, 4.8 Hz, 1H), 1.10-0.97 (m, 1H), $0.76(\mathrm{~s}, 9 \mathrm{H}), 0.54(\mathrm{t}, J=5.1 \mathrm{~Hz}, 1 \mathrm{H}), 0.21-0.12(\mathrm{~m}, 1 \mathrm{H})$, $0.08(\mathrm{~s}, 3 \mathrm{H}),-0.25(\mathrm{~s}, 3 \mathrm{H}) ;{ }^{13} \mathrm{C} \mathrm{NMR}\left(75 \mathrm{MHz}, \mathrm{CDCl}_{3}\right) \delta 140.5,139.7,130.0,128.7,128.3$, 
126.3, 59.6, 30.6, 27.1, 25.9, 25.2, 22.1, 20.0, 18.1, -3.2, -3.4; Anal. Cacld. for $\mathrm{C}_{18} \mathrm{H}_{28} \mathrm{OSi}: \mathrm{C}$, 74.94; H, 9.78; Found: C, 75.07; H, 9.91.

\section{Table 1, Entry 6}

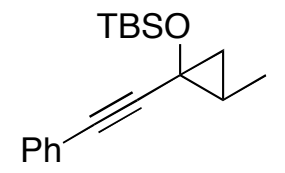

Colorless liquid; $[\alpha]^{20}{ }_{\mathrm{D}}=+42.8\left(c 1.56, \mathrm{CHCl}_{3}\right)\left(86 \%\right.$ ee); IR (neat) $2230 \mathrm{~cm}^{-1} ;{ }^{1} \mathrm{H}$ NMR $(300$ $\left.\mathrm{MHz}, \mathrm{CDCl}_{3}\right) \delta$ 7.41-7.37 (m, 2H), 7.32-7.28 (m, 3H), 1.25-1.17 (m, 5H), 0.92 (s, 9H), 0.60-0.56 $(\mathrm{m}, 1 \mathrm{H}), 0.29(\mathrm{~s}, 3 \mathrm{H}), 0.26(\mathrm{~s}, 3 \mathrm{H}) ;{ }^{13} \mathrm{C} \mathrm{NMR}\left(75 \mathrm{MHz}, \mathrm{CDCl}_{3}\right) \delta 131.4,128.4,128.0,123.5$, 93.2, 81.8, 49.8, 26.1, 24.7, 22.5, 18.4, 12.7, -3.4, -3.8; Anal. Cacld. for $\mathrm{C}_{18} \mathrm{H}_{26} \mathrm{OSi}$ : C, 75.46; H, 9.15; Found: C, 75.40; H, 8.89.

Iwasawa, N.; Matsuo, T.; Iwamoto, M.; Ikeno, T. J. Am. Chem. Soc, 1998, 120, 3903.

\section{Table 1, Entry 7}

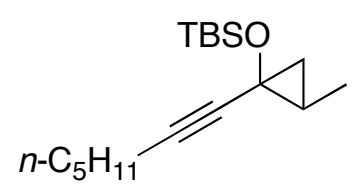

Colorless liquid; $[\alpha]^{20}{ }_{D}=+23.4\left(c 1.45, \mathrm{CHCl}_{3}\right)(86 \%$ ee $)$; IR (neat) $1472,1247 \mathrm{~cm}^{-1} ;{ }^{1} \mathrm{H} \mathrm{NMR}$ $\left(300 \mathrm{MHz}, \mathrm{CDCl}_{3}\right) \delta 2.18(\mathrm{t}, J=6.9 \mathrm{~Hz}, 2 \mathrm{H}), 1.52-1.45(\mathrm{~m}, 2 \mathrm{H}), 1.41-1.28(\mathrm{~m}, 4 \mathrm{H}), 1.16-1.00$ (m, 5H), $0.90(\mathrm{t}, J=6.9 \mathrm{~Hz}, 3 \mathrm{H}$, $) 0.89(\mathrm{~s}, 9 \mathrm{H}), 0.42(\mathrm{t}, J=3.9 \mathrm{~Hz}, 1 \mathrm{H}), 0.23(\mathrm{~s}, 3 \mathrm{H}), 0.20(\mathrm{~s}, 3 \mathrm{H})$; ${ }^{13} \mathrm{C}$ NMR $\left(75 \mathrm{MHz}, \mathrm{CDCl}_{3}\right) \delta 83.7,82.0,49.7,31.4,28.7,26.1,24.2,22.5,21.9,19.1,18.3,14.3$, 12.6, -3.4, -3.9; Anal. Cacld. for $\mathrm{C}_{17} \mathrm{H}_{32} \mathrm{OSi}$ C, 72.79; H, 11.50; Found: C, 72.70; H, 11.52 .

\section{Table 1, Entry 8}

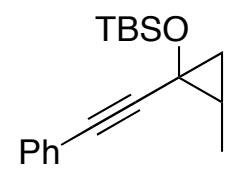


Colorless liquid; $[\alpha]^{20}{ }_{\mathrm{D}}=-11.0\left(c\right.$ 1.38, $\left.\mathrm{CHCl}_{3}\right)(93 \%$ ee $) ; \mathrm{IR}$ (neat) $1218 \mathrm{~cm}^{-1} ;{ }^{1} \mathrm{H}$ NMR $(300$ $\left.\mathrm{MHz}, \mathrm{CDCl}_{3}\right) \delta$ 7.43-7.39 (m 2H), 7.32-7.28 (m, 3H), 1.28-1.17 (m, 5H), 0.90 (s, 9H), 0.68 (dd, $J=5.4,3.6 \mathrm{~Hz}, 1 \mathrm{H}), 0.25(\mathrm{~s}, 3 \mathrm{H}), 0.24(\mathrm{~s}, 3 \mathrm{H}) ;{ }^{13} \mathrm{C} \mathrm{NMR}\left(75 \mathrm{MHz}, \mathrm{CDCl}_{3}\right) \delta 131.5,128.4$, 128.0, 123.5, 90.0, 84.8, 52.5, 26.0, 25.3, 23.1, 18.0, 15.4, -3.6; Anal. Cacld. for $\mathrm{C}_{18} \mathrm{H}_{26} \mathrm{OSi}$ : C, 75.46; H, 9.15; Found: C, 75.64; H, 8.83.

Iwasawa, N.; Matsuo, T.; Iwamoto, M.; Ikeno, T. J. Am. Chem. Soc, 1998, 120, 3903.

\section{Table 1, Entry 9}

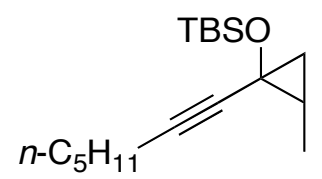

Colorless liquid; $[\alpha]^{20}{ }_{\mathrm{D}}=-3.6\left(c\right.$ 1.37, $\left.\mathrm{CHCl}_{3}\right)\left(93 \%\right.$ ee); IR (neat) $2237 \mathrm{~cm}^{-1} ;{ }^{1} \mathrm{H}$ NMR (300 $\left.\mathrm{MHz}, \mathrm{CDCl}_{3}\right) \delta 2.22(\mathrm{t}, J=6.9 \mathrm{~Hz}, 2 \mathrm{H}), 1.56-1.47(\mathrm{~m}, 2 \mathrm{H}), 1.43-1.26(\mathrm{~m}, 4 \mathrm{H}), 1.10(\mathrm{~d}, J=2.4$ Hz, 3H), 1.09-1.01 (m, 2H), $0.90(\mathrm{t}, J=6.9 \mathrm{~Hz}, 3 \mathrm{H}), 0.86(\mathrm{~s}, 9 \mathrm{H}),, 0.48-0.45(\mathrm{~m}, 1 \mathrm{H}), 0.19$ (s, 3H), $0.18(\mathrm{~s}, 3 \mathrm{H}) ;{ }^{13} \mathrm{C}$ NMR $\left(75 \mathrm{MHz}, \mathrm{CDCl}_{3}\right) \delta 85.1,80.3,52.3,31.3,28.8,26.0,24.6,22.5$, 22.2, 19.1, 18.0, 15.3, 14.3, -3.7; Anal. Cacld. for $\mathrm{C}_{17} \mathrm{H}_{32} \mathrm{OSi}$ : C, 72.79; H, 11.50; Found: C, $72.96 ; \mathrm{H}, 11.58$.

\section{Table 1, Entry 10}<smiles>CS(=O)(=O)OC12C=CCCC1C2</smiles>

Colorless liquid; $[\alpha]^{20}{ }_{\mathrm{D}}=-75.1\left(c 1.29, \mathrm{CHCl}_{3}\right)\left(92 \%\right.$ ee); IR (neat) $1456 \mathrm{~cm}^{-1} ;{ }^{1} \mathrm{H}$ NMR (300 $\left.\mathrm{MHz}, \mathrm{CDCl}_{3}\right) \delta 6.12(\mathrm{~d}, J=10.2 \mathrm{~Hz}, 1 \mathrm{H}), 5.37(\mathrm{dd}, J=10.2,7.8 \mathrm{~Hz}, 1 \mathrm{H}), 2.01-1.92(\mathrm{~m}, 1 \mathrm{H})$, $1.88-1.78(\mathrm{~m}, 1 \mathrm{H}), 1.66-1.53(\mathrm{~m}, 2 \mathrm{H}), 1.52-1.46(\mathrm{~m}, 1 \mathrm{H}), 0.98(\mathrm{dd}, J=9.9,5.1 \mathrm{~Hz}, 1 \mathrm{H}), 0.76(\mathrm{t}$, 
$J=6.0 \mathrm{~Hz}, 1 \mathrm{H}), 0.16(\mathrm{~s}, 9 \mathrm{H}) ;{ }^{13} \mathrm{C} \mathrm{NMR}\left(75 \mathrm{MHz}, \mathrm{CDCl}_{3}\right) \delta 133.6,121.6,54.3,23.4,20.6,18.2$, $17.8,1.5$.

Rinderhagen, H.; Mattay, J. Chem. Eur. J. 2004, 10, 851.

\section{Table 1, Entry 11}

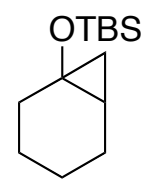

Colorless liquid; $[\alpha]^{20}{ }_{\mathrm{D}}=+14.1\left(c 0.61, \mathrm{CHCl}_{3}\right)\left(72 \%\right.$ ee); IR (neat) $1471 \mathrm{~cm}^{-1} ;{ }^{1} \mathrm{H}$ NMR $(300$ $\left.\mathrm{MHz}, \mathrm{CDCl}_{3}\right) \delta 2.16-2.08(\mathrm{~m}, 1 \mathrm{H}), 2.05-1.96(\mathrm{~m}, 1 \mathrm{H}), 1.93-1.83(\mathrm{~m}, 1 \mathrm{H}), 1.56-1.45(\mathrm{~m}, 1 \mathrm{H})$, $1.39(\mathrm{dd}, J=13.8,6.9 \mathrm{~Hz}, 1 \mathrm{H}), 1.29-1.20(\mathrm{~m}, 2 \mathrm{H}), 1.14-0.99(\mathrm{~m}, 2 \mathrm{H}), 0.86(\mathrm{~s}, 9 \mathrm{H}), 0.82-0.76$ $(\mathrm{m}, 1 \mathrm{H}), 0.30(\mathrm{t}, J=5.7 \mathrm{~Hz}, 1 \mathrm{H}), 0.11(\mathrm{~s}, 6 \mathrm{H}) ;{ }^{13} \mathrm{C} \mathrm{NMR}\left(75 \mathrm{MHz}, \mathrm{CDCl}_{3}\right) \delta 56.4,32.7,26.1$, 24.9, 22.2, 21.8, 19.8, 19.1, 18.0, -3.0, -3.1; Anal. Cacld. for $\mathrm{C}_{13} \mathrm{H}_{26} \mathrm{OSi}$ : C, 68.96; H, 11.57; Found: C, 69.20; H, 11.39.

(a) Ikura, K.; Ryu, I.; Ogawa, A.; Kambe, N.; Sonoda, N. Tetrahedron Lett. 1989, 30, 6887. (b) Ikura, K.; Ryu, I.; Kambe, N. Sonoda, N. J. Am. Chem. Soc. 1992, 114, 1520.

\section{Table 1, Entry 12}

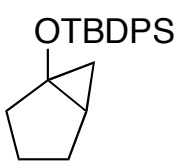

Colorless liquid; $[\alpha]^{20}=+3.9\left(c 1.21, \mathrm{CHCl}_{3}\right)\left(89 \%\right.$ ee); IR (neat) $1473,1428,1360 \mathrm{~cm}^{-1} ;{ }^{1} \mathrm{H}$ NMR (300 MHz, $\left.\mathrm{CDCl}_{3}\right) \delta$ 7.72-7.67 (m, 4H), 7.45-7.34 (m, 6H), 1.90-1.70 (m, 2H), 1.55-1.19 $(\mathrm{m}, 4 \mathrm{H}), 1.04(\mathrm{~s}, 9 \mathrm{H}), 0.95-0.83(\mathrm{~m}, 1 \mathrm{H}), 0.77(\mathrm{ddd}, J=9.3,6.0,1.5 \mathrm{~Hz}, 1 \mathrm{H}), 0.36(\mathrm{t}, J=5.1 \mathrm{~Hz}$, $1 \mathrm{H}) ;{ }^{13} \mathrm{C} \mathrm{NMR}\left(75 \mathrm{MHz}, \mathrm{CDCl}_{3}\right) \delta 135.9,129.6,129.5,127.5,66.6,34.0,27.2,26.6,24.2,21.2$, 19.2, 15.0; HRMS cacld. for $\mathrm{C}_{22} \mathrm{H}_{28} \mathrm{OSi}\left(\mathrm{M}^{+}\right)$: 336.1909; Found: 336.1900 . 
The chromatograms for the determination of enantiomeric excess of cyclopropanes (Table 1)

Table 1, Entry 1<smiles>COC(C)(c1ccccc1)C1CC1C</smiles>

GC Conditions: Column: Chiraldex B-DM (Cat No. 77023), Advanced Separation Technologies, Inc.. Oven: $85^{\circ} \mathrm{C}$; Carrier: Helium, head pressure 30 psi; Detection: FID $250^{\circ} \mathrm{C}$

Racemate
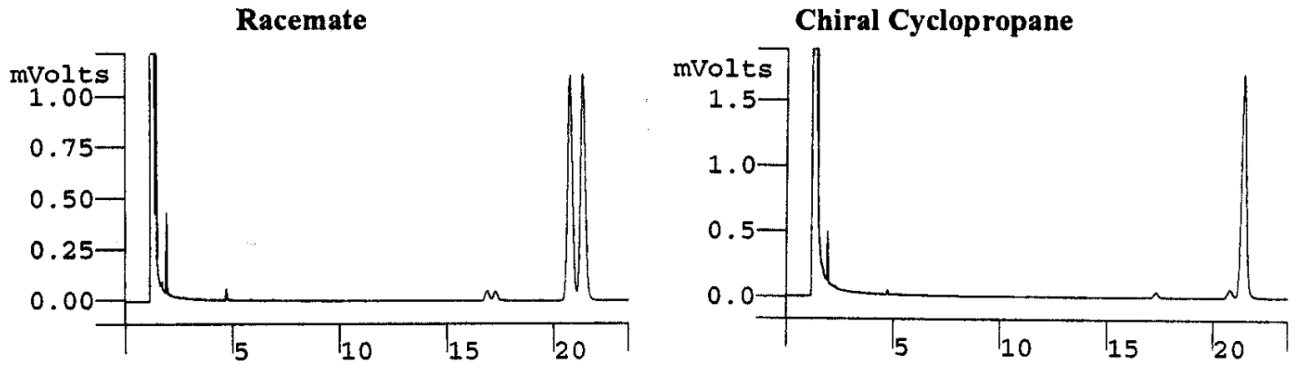

\begin{tabular}{cccc}
$\begin{array}{c}\text { Peak } \\
\text { No }\end{array}$ & $\begin{array}{c}\text { Peak } \\
\text { Name }\end{array}$ & $\begin{array}{c}\text { Ret. Time } \\
\text { (min) }\end{array}$ & \multicolumn{1}{c}{$\begin{array}{c}\text { Minutes } \\
\text { Result }()\end{array}$} \\
\hline 1 & & 20.771 & 49.6259 \\
2 & $\frac{21.371}{\text { Totals }}$ & 50.3741 \\
\hline $\mathbf{1 0 0 . 0 0 0 0}$
\end{tabular}

\begin{tabular}{|c|c|c|c|}
\hline $\begin{array}{c}\text { Peak } \\
\text { No }\end{array}$ & $\begin{array}{c}\text { Peak } \\
\text { Name }\end{array}$ & $\begin{array}{l}\text { Ret. Time } \\
\text { (min) }\end{array}$ & $\begin{array}{l}\text { Minutes } \\
\text { Result () }\end{array}$ \\
\hline 1 & & 20.756 & 3.3818 \\
\hline 2 & & 21.386 & 96.6182 \\
\hline
\end{tabular}

Table 1, Entry 2<smiles>COc1ccc(C2(OC)CCC2C)cc1</smiles>

GC Conditions: Column: Chiraldex B-DM (Cat No. 77023), Advanced Separation Technologies, Inc.. Oven: $70^{\circ} \mathrm{C}$; Carrier: Helium, head pressure 30 psi; Detection: FD $250^{\circ} \mathrm{C}$

Racemate

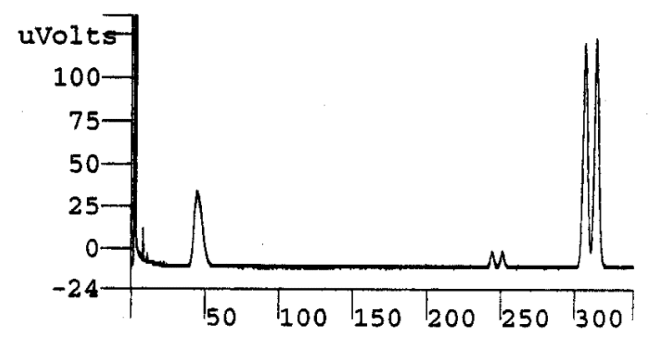

\begin{tabular}{|c|c|c|c|}
\hline $\begin{array}{c}\text { Peak } \\
\text { No }\end{array}$ & $\begin{array}{l}\text { Peak } \\
\text { Name }\end{array}$ & $\begin{array}{c}\text { Ret. Time } \\
\text { (min) }\end{array}$ & $\begin{array}{l}\text { Minutes } \\
\text { Result () }\end{array}$ \\
\hline 1 & & 306.631 & 50.2149 \\
\hline \multirow[t]{2}{*}{2} & & 314.163 & 49.7851 \\
\hline & otals & & 100.0000 \\
\hline
\end{tabular}

Chiral Cyclopropane

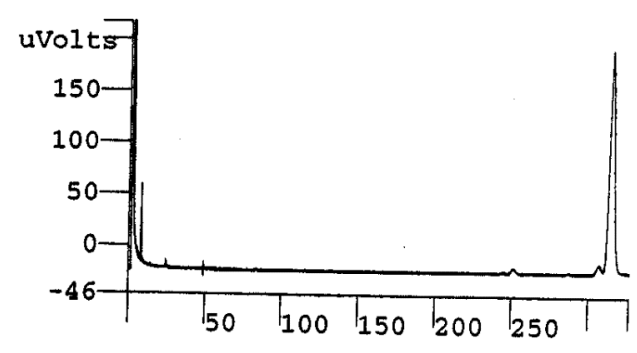

\begin{tabular}{|c|c|c|c|}
\hline $\begin{array}{c}\text { Peak } \\
\text { No }\end{array}$ & $\begin{array}{c}\text { Peak } \\
\text { Name }\end{array}$ & $\begin{array}{l}\text { Ret. Time } \\
\text { (min) }\end{array}$ & $\begin{array}{l}\text { Minutes } \\
\text { Result () }\end{array}$ \\
\hline 1 & & 306.521 & 3.2779 \\
\hline 2 & & 314.563 & 96.7221 \\
\hline
\end{tabular}


Table 1, Entry 3<smiles>COC1(c2ccc3ccccc3c2)CC1C</smiles>

HIPLC Conditions: Column: Chiralcel OD (Column No. OD00CE-DL010), Chiral Technologies, Inc. Eluent: Hexanes/IPA (99/1); Flow Rate: $1.0 \mathrm{~mL} / \mathrm{min}$; Detection: UV254

Racemate

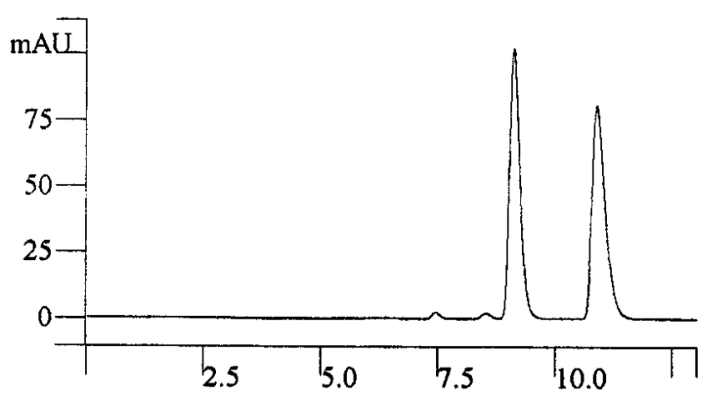

Minutes

\begin{tabular}{|c|c|c|c|}
\hline $\begin{array}{c}\text { Peak } \\
\text { No }\end{array}$ & $\begin{array}{l}\text { Peak } \\
\text { Name }\end{array}$ & $\begin{array}{l}\text { Ret. } \\
\text { Time } \\
\text { (min) }\end{array}$ & Result 0 \\
\hline 1 & & 9.104 & 49.6770 \\
\hline 2 & & 10876 & 50.3230 \\
\hline
\end{tabular}

Chiral Cyclopropane

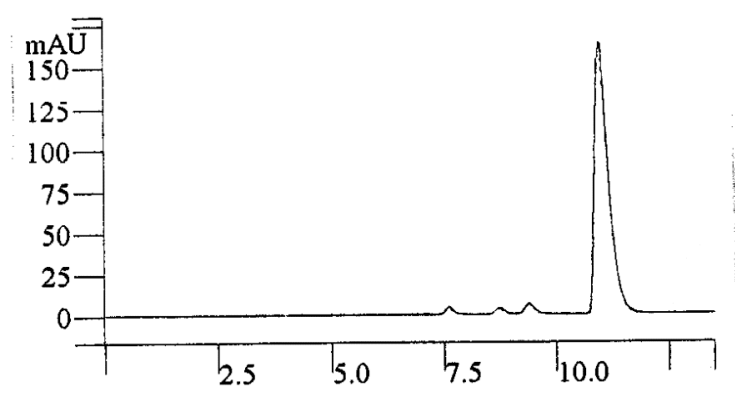

Minutes

\begin{tabular}{|c|c|c|c|}
\hline $\begin{array}{c}\text { Peak } \\
\text { No }\end{array}$ & $\begin{array}{l}\text { Peak } \\
\text { Name }\end{array}$ & $\begin{array}{l}\text { Ret. } \\
\text { Time } \\
\text { (min) }\end{array}$ & Result 0 \\
\hline 1 & & 9.377 & 2.5251 \\
\hline 2 & - & 10.954. & 97.4749 \\
\hline
\end{tabular}

Table 1, Entry 4<smiles></smiles>

GC Conditions: Column: Chiraldex B-DM (Cat No. 77023), Advanced Separation Technologies, Inc. Oven: $140^{\circ} \mathrm{C}$; Carrier: Helium, head pressure 30 psi; Detection: $\mathrm{FD} 250^{\circ} \mathrm{C}$ Racemate

Chiral Cyclopropane
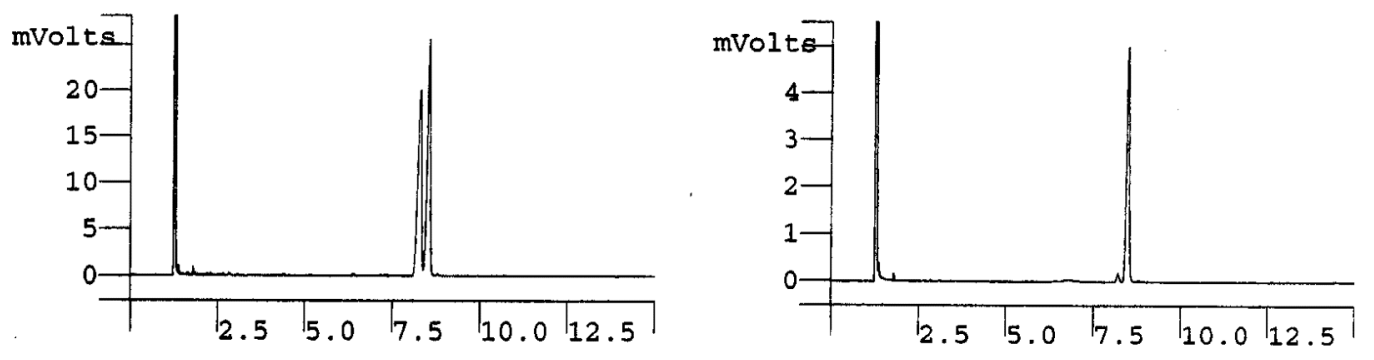

\begin{tabular}{|c|c|c|c|}
\hline $\begin{array}{c}\text { Peak } \\
\text { No }\end{array}$ & $\begin{array}{c}\text { Peak } \\
\text { Name }\end{array}$ & $\begin{array}{l}\text { Ret. Time } \\
\text { (min) }\end{array}$ & $\begin{array}{l}\text { Minutes } \\
\text { Result () }\end{array}$ \\
\hline 1 & & 8.296 & 49.8483 \\
\hline 2 & & 8.552 & 50.1517 \\
\hline & Totals & & 100.0000 \\
\hline
\end{tabular}

\begin{tabular}{|c|c|c|c|}
\hline $\begin{array}{c}\text { Peak } \\
\text { No }\end{array}$ & $\begin{array}{l}\text { Peak } \\
\text { Name }\end{array}$ & $\begin{array}{l}\text { Ret. Time } \\
\text { (min) }\end{array}$ & $\begin{array}{l}\text { Minutes } \\
\text { Result } 0\end{array}$ \\
\hline 1 & & 8.201 & 3.5868 \\
\hline 2 & & 8.472 & 96.4132 \\
\hline & Totals & & 100.0000 \\
\hline
\end{tabular}


Table 1, Entry 5<smiles>OC12CC1CCCc1ccccc12</smiles>

GC Conditions: Column: Chiraldex B-DM (Cat No. 77023), Advanced Separation Technologies, Inc.. Oven: $140^{\circ} \mathrm{C}$; Carrier: Helium, head pressure 30 psi; Detection: FID $250^{\circ} \mathrm{C}$

Racemate

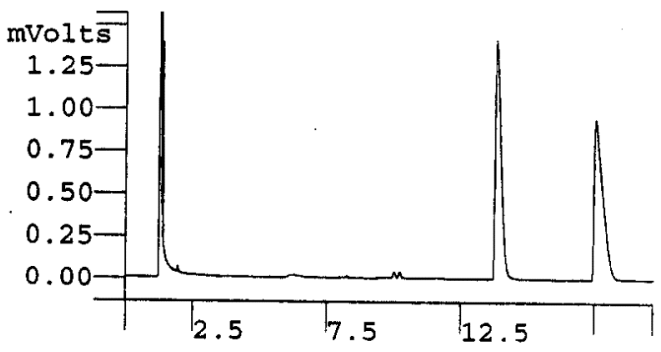

Minutes

\begin{tabular}{|c|c|c|c|}
\hline $\begin{array}{c}\text { Peak } \\
\text { No }\end{array}$ & $\begin{array}{l}\text { Peak } \\
\text { Name }\end{array}$ & $\begin{array}{l}\text { Ret. Time } \\
\text { (min) }\end{array}$ & Result () \\
\hline 1 & & 13.778 & 49.9359 \\
\hline \multirow[t]{2}{*}{2} & & 17.519 & 50.0641 \\
\hline & Totals & & 100.0000 \\
\hline
\end{tabular}

\section{Chiral Cyclopropane}

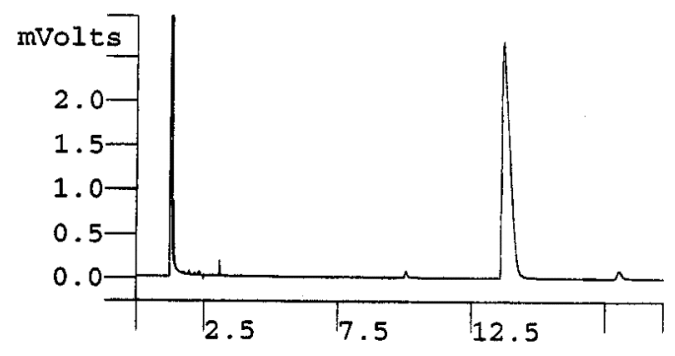

\begin{tabular}{|c|c|c|c|}
\hline $\begin{array}{c}\text { Peak } \\
\text { No }\end{array}$ & $\begin{array}{l}\text { Peak } \\
\text { Name }\end{array}$ & $\begin{array}{l}\text { Ret. Time } \\
\text { (min) }\end{array}$ & $\begin{array}{l}\text { Minutes } \\
\text { Result () }\end{array}$ \\
\hline 1 & & 13.654 & 97.9801 \\
\hline 2 & & 18.004 & 2.0199 \\
\hline
\end{tabular}

Table 1, Entry 6<smiles>CC1CC1(O)C#Cc1ccccc1</smiles>

GC Conditions: Column: Chiraldex B-DM (Cat No. 77023), Advanced Separation Technologies, Inc.. Oven: $120^{\circ} \mathrm{C}$; Carrier: Helium, head pressure 30 psi; Detection: FID $250^{\circ} \mathrm{C}$

Racemate

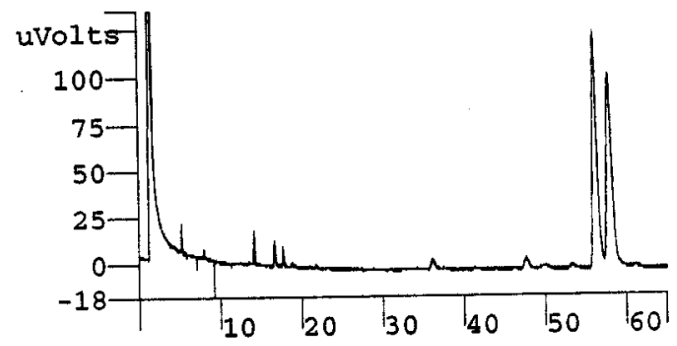

Minutes

\begin{tabular}{|c|c|c|c|}
\hline $\begin{array}{c}\text { Peak } \\
\text { No }\end{array}$ & $\begin{array}{l}\text { Peak } \\
\text { Name }\end{array}$ & $\begin{array}{l}\text { Ret. Time } \\
\text { (min) }\end{array}$ & Result () \\
\hline 1 & & 56.039 & 50.0781 \\
\hline 2 & & 57.827 & 49.9219 \\
\hline & Total & & 100.0000 \\
\hline
\end{tabular}

Chiral Cyclopropane

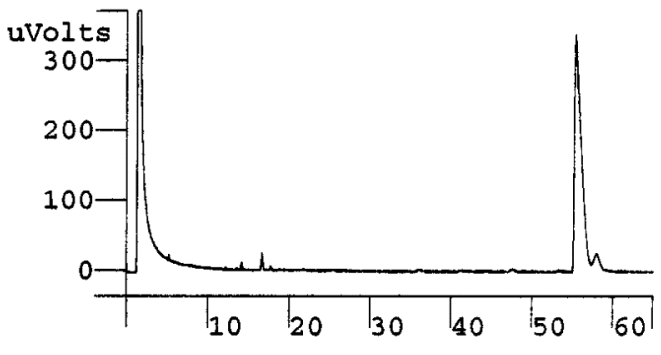

Minutes

\begin{tabular}{|c|c|c|c|}
\hline $\begin{array}{c}\text { Peak } \\
\text { No }\end{array}$ & $\begin{array}{l}\text { Peak } \\
\text { Name }\end{array}$ & $\begin{array}{l}\text { Ret. Time } \\
\text { (min) }\end{array}$ & Result () \\
\hline 1 & & 55.364 & 93.1798 \\
\hline 2 & & 57.908 & 6.8202 \\
\hline & Total & & 100.0000 \\
\hline
\end{tabular}


Table 1, Entry 7

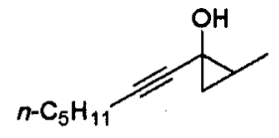

GC Conditions: Column: Chiraldex B-DM (Cat No. 77023), Advanced Separation Technologies, Inc..

Oven: $100^{\circ} \mathrm{C}$; Carrier: Helium, head pressure 30 psi; Detection: FID $250^{\circ} \mathrm{C}$

Racemate

Chiral Cyclopropane
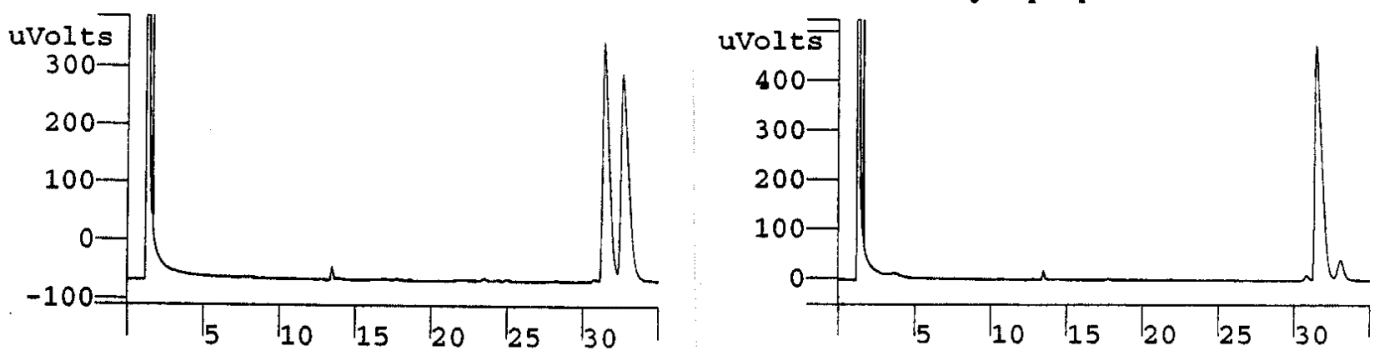

\begin{tabular}{|c|c|c|c|}
\hline $\begin{array}{c}\text { Peak } \\
\text { No }\end{array}$ & $\begin{array}{l}\text { Peak } \\
\text { Name }\end{array}$ & $\begin{array}{l}\text { Ret. Time } \\
\text { (min) }\end{array}$ & $\begin{array}{l}\text { Minutes } \\
\text { Result () }\end{array}$ \\
\hline 1 & & 31.313 & 49.6698 \\
\hline \multirow[t]{2}{*}{2} & & 32.531 & 50.3302 \\
\hline & Totals & & 100.0000 \\
\hline
\end{tabular}

\begin{tabular}{|c|c|c|c|}
\hline $\begin{array}{c}\text { Peak } \\
\text { No }\end{array}$ & $\begin{array}{c}\text { Peak } \\
\text { Name }\end{array}$ & $\begin{array}{l}\text { Ret. Time } \\
\text { (min) }\end{array}$ & $\begin{array}{l}\text { Minutes } \\
\text { Result () }\end{array}$ \\
\hline 1 & . & 31.391 & 92.7614 \\
\hline 2 & & 32.998 & 7.2386 \\
\hline & Totals & & 100.0000 \\
\hline
\end{tabular}

Table 1, Entry 8<smiles>CC1CC1(O)C#Cc1ccccc1</smiles>

GC Conditions: Column: Chiraldex B-DM (Cat No. 77023), Advanced Separation Technologies, Inc.. Oven: $120^{\circ} \mathrm{C}$; Carrier: Helium, head pressure 30 psi; Detection: FID $250^{\circ} \mathrm{C}$

Racemate

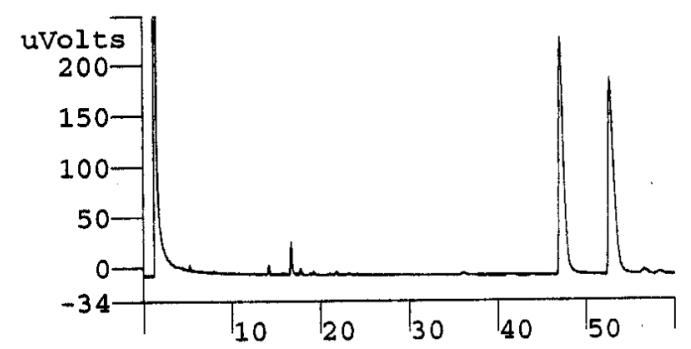

Minutes

\begin{tabular}{cccc}
$\begin{array}{c}\text { Peak } \\
\text { No }\end{array}$ & $\begin{array}{c}\text { Peak } \\
\text { Name }\end{array}$ & $\begin{array}{c}\text { Ret. Time } \\
\text { (min) }\end{array}$ & Result () \\
\hline 1 & & 47.088 & 50.3874 \\
2 \\
\hline Totals & 52.669 & $\frac{49.6126}{100.0000}$
\end{tabular}

Chiral Cyclopropane

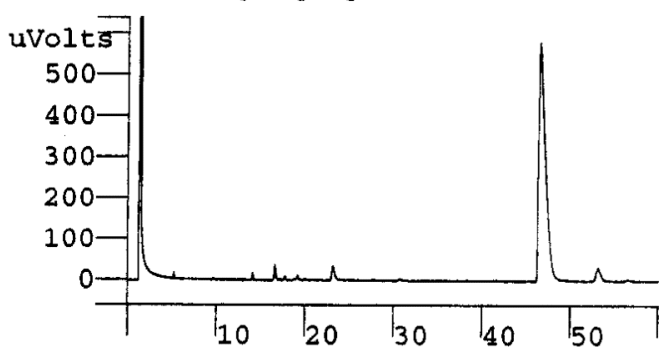

\begin{tabular}{|c|c|c|c|}
\hline $\begin{array}{c}\text { Peak } \\
\text { No }\end{array}$ & $\begin{array}{c}\text { Peak } \\
\text { Name }\end{array}$ & $\begin{array}{c}\text { Ret. Time } \\
\text { (min) }\end{array}$ & $\begin{array}{l}\text { Minutes } \\
\text { Result 0 }\end{array}$ \\
\hline 1 & & 46.429 & 96.3546 \\
\hline 2 & & 53.099 & 3.6454 \\
\hline & Totals & & 100.0000 \\
\hline
\end{tabular}


Table 1, Entry 9

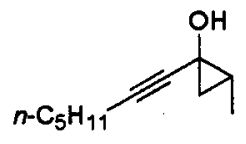

GC Conditions: Column: Chiraldex B-DM (Cat No. 77023), Advanced Separation Technologies, Inc..

Oven: $90^{\circ} \mathrm{C}$; Carrier: Helium, head pressure 30 psi; Detection: FID $250^{\circ} \mathrm{C}$

Racemate

Chiral Cyclopropane
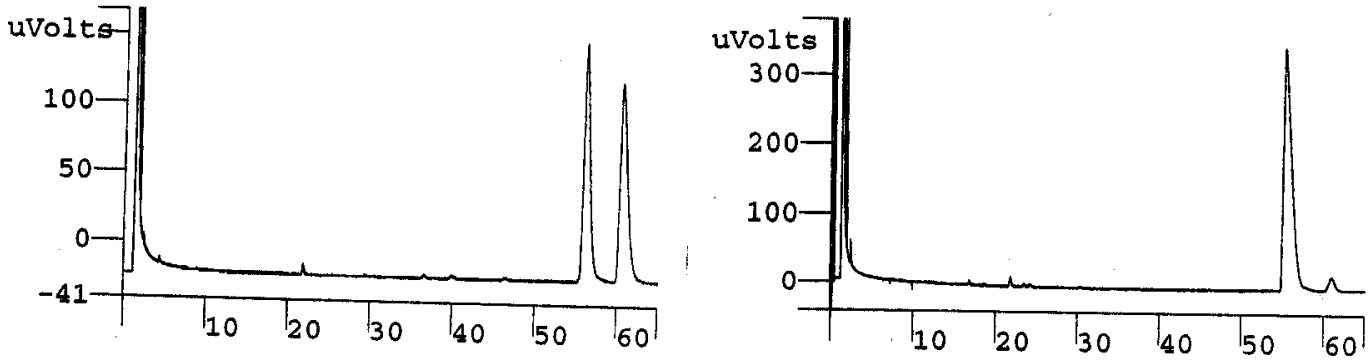

\begin{tabular}{|c|c|c|c|}
\hline $\begin{array}{c}\text { Peak } \\
\text { No }\end{array}$ & $\begin{array}{c}\text { Peak } \\
\text { Name }\end{array}$ & $\underset{\text { (min) }}{\text { Ret. Time }}$ & $\begin{array}{l}\text { Minutes } \\
\text { Result 0 }\end{array}$ \\
\hline 1 & & 55.889 & 50.1724 \\
\hline 2 & & 60.279 & 49.8276 \\
\hline
\end{tabular}

\begin{tabular}{|c|c|c|c|}
\hline $\begin{array}{c}\text { Peak } \\
\text { No }\end{array}$ & $\begin{array}{c}\text { Peak } \\
\text { Name }\end{array}$ & $\underset{\text { (min) }}{\text { Ret. Time }}$ & $\begin{array}{l}\text { Minutes } \\
\text { Result () }\end{array}$ \\
\hline 1 & & 55.042 & 96.2806 \\
\hline 2 & & 60.831 & 3.7194 \\
\hline
\end{tabular}

Table 1, Entry 10<smiles>COC12C=CCCC1C2</smiles>

GC Conditions: Column: Chiraldex B-DM (Cat No. 77023), Advanced Separation Technologies, Inc. Oven: $70^{\circ} \mathrm{C}$; Carrier: Helium, head pressure $30 \mathrm{psi}$; Detection: FID $250^{\circ} \mathrm{C}$

Racemate

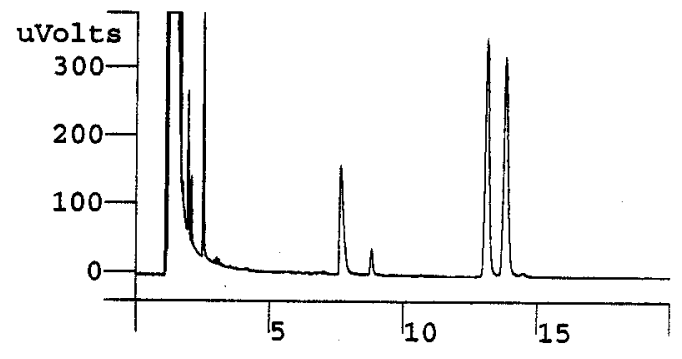

Minutes

\begin{tabular}{|c|c|c|c|}
\hline $\begin{array}{c}\text { Peak } \\
\text { No }\end{array}$ & $\begin{array}{c}\text { Peak } \\
\text { Name }\end{array}$ & $\begin{array}{l}\text { Ret. Time } \\
\text { (min) }\end{array}$ & Result () \\
\hline 1 & & 13.097 & 49.7521 \\
\hline 2 & & 13.792 & 50.2479 \\
\hline & Totals & & 100.0000 \\
\hline
\end{tabular}

Chiral Cyclopropane

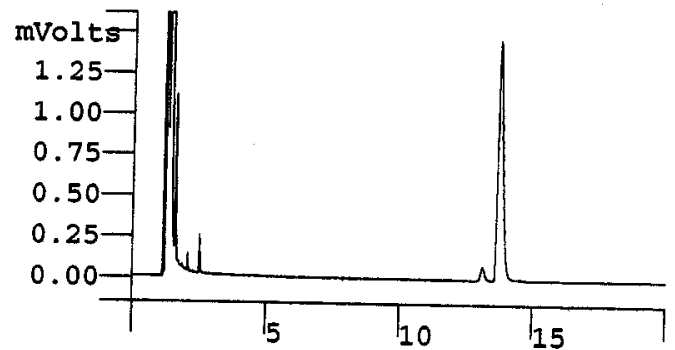

Minutes

Peak Peak Ret. Time Result () No Name (min)

\begin{tabular}{|c|c|c|}
\hline 1 & 13.118 & 4.1404 \\
\hline 2 & 13.698 & 95.8596 \\
\hline
\end{tabular}


Table 1, Entry 11<smiles>OC12CCCCC1C2</smiles>

GC Conditions: Column: Chiraldex B-DM (Cat No. 77023), Advanced Separation Technologies, Inc.. Oven: $50^{\circ} \mathrm{C}$; Carrier: Helium, head pressure 20 psi; Detection: $\mathrm{FD} 200^{\circ} \mathrm{C}$

Racemate

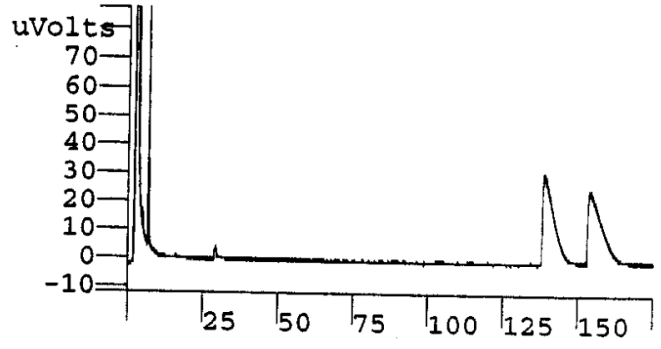

\begin{tabular}{cccc}
$\begin{array}{c}\text { Peak } \\
\text { No }\end{array}$ & $\begin{array}{c}\text { Peak } \\
\text { Name }\end{array}$ & $\begin{array}{c}\text { Ret. Time } \\
\text { (min) }\end{array}$ & \multicolumn{1}{c}{$\begin{array}{c}\text { Minutes } \\
\text { Result }()\end{array}$} \\
\hline 1 & & 138.291 & 49.9608 \\
\hline 2 & & 153.546 & 50.0392 \\
\hline & & 100.0000
\end{tabular}

Chiral Cyclopropane

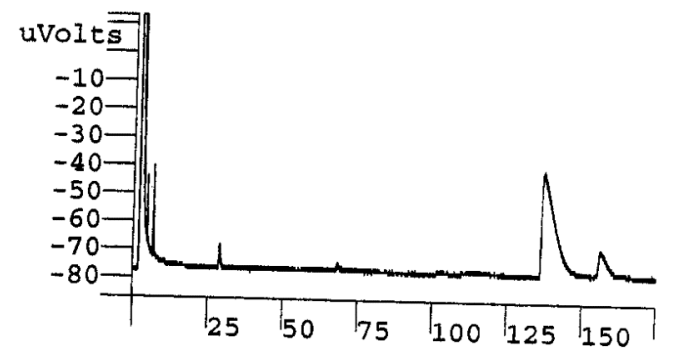

\begin{tabular}{|c|c|c|c|}
\hline $\begin{array}{c}\text { Peak } \\
\text { No }\end{array}$ & $\begin{array}{c}\text { Peak } \\
\text { Name }\end{array}$ & $\begin{array}{c}\text { Ret. Time } \\
\text { (min) }\end{array}$ & $\begin{array}{l}\text { Minutes } \\
\text { Result (O }\end{array}$ \\
\hline 1 & & .136 .989 & 85.8947 \\
\hline 2 & & 155.883 & 14.1053 \\
\hline & Totals & & 100.0000 \\
\hline
\end{tabular}

Table 1, Entry 12

$\mathrm{HO}$<smiles>CC12CCCC1C2</smiles>

GC Conditions: Column: Chiraldex B-DM (Cat No. 77023), Advanced Separation Technologies, Inc.. Oven: $50^{\circ} \mathrm{C}$; Carrier: Helium, head pressure 20 psi; Detection: FID $200^{\circ} \mathrm{C}$

Racemate

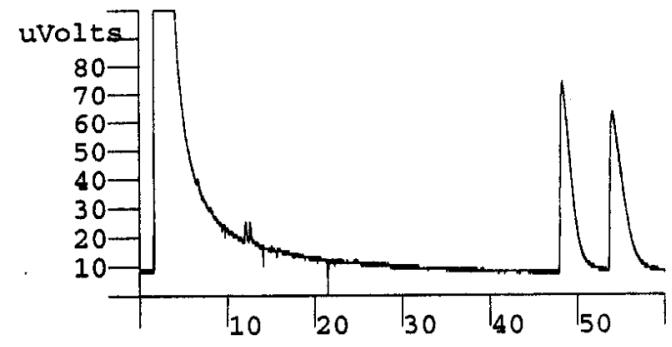

Minutes

\begin{tabular}{|c|c|c|c|}
\hline $\begin{array}{c}\text { Peak } \\
\text { No }\end{array}$ & $\begin{array}{l}\text { Peak } \\
\text { Name }\end{array}$ & $\begin{array}{l}\text { Ret. Time } \\
\text { (min) }\end{array}$ & Result 0 \\
\hline 1 & & 48.292 & 49.9713 \\
\hline 2 & & 54.046 & 50.0287 \\
\hline & Totals & & 100.0000 \\
\hline
\end{tabular}

Chiral Cyclopropane

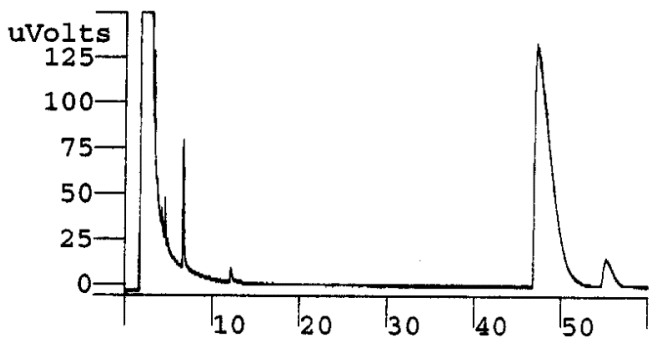

\begin{tabular}{|c|c|c|c|}
\hline $\begin{array}{c}\text { Peak } \\
\text { No }\end{array}$ & $\begin{array}{c}\text { Peak } \\
\text { Name }\end{array}$ & $\begin{array}{l}\text { Ret. Time } \\
\text { (min) }\end{array}$ & $\begin{array}{l}\text { Minutes } \\
\text { Result () }\end{array}$ \\
\hline 1 & & 47.042 & 94.3275 \\
\hline 2 & & 55.134 & 5.6725 \\
\hline
\end{tabular}

